\title{
Von der Prophylaxe bis zur unterstützenden Parodontitis-Therapie (UPT)
}

\begin{abstract}
Es sind zwei Dinge, die Patienten nach einer geschlossenen Parodontitis-Therapie als besonders unangenehm beschreiben: die Injektionen und das oft über mehrere Stunden anhaltende Taubheitsgefühl. Mit der subgingivalen Applikation von Dynexan Mundge $^{\circledR}$ aus Zylinderampullen treten genau diese Probleme meist erst gar nicht auf, bei gleichzeitig guter Anästhesie-Wirkung. Diese guten Erfahrungen erhöhen auch die Compliance der Patienten in der anschließenden Betreuungsphase.
\end{abstract}

$\mathrm{M}$ it den modernen, minimalinvasiven Methoden und der Anästhesie ohne Spritze schaffen wir die Voraussetzungen, dass auch ängstliche Patienten der Behandlung zustimmen. Schmerzfrei von Anfang an, dann folgen Ihre Patienten dem Praxiskonzept auch langfristig im Recall.

\section{Anästhesie ohne Injektion}

Auch tiefere Taschen ab $5 \mathrm{~mm}$ lassen sich mit der subgingivalen Applikation von Dynexan Mundgel $^{\circ}$ aus Zylinderampullen fast immer sicher betäuben. Da eine längere Wartezeit auf den Wirkungseintritt entfällt, ist der Zeitaufwand vergleichbar mit der Infiltrationsanästhesie. Die Wirkungsdauer reicht problemlos für die Behandlung eines Quadranten, meist kann ein gesamter Kiefer in einem Zug behandelt werden. Die gesetzlichen Krankenkassen akzeptieren eine Behandlung ohne Infiltrations- oder Leitungsanästhesie, ein Hinweis auf eine private Leistung kann hilfreich sein. Die Abrechnung erfolgt privat, da die Berechnung als bloße Oberflächenanästhesie nicht angemessen wäre. Sie finden weitere Informationen und Abrechnungshinweise in der Beratungsmappe, die Sie bei Kreussler Pharma jederzeit anfordern können. Die Patienten schätzen den Komfortgewinn der gut verträglichen Betäubung ohne Injektion durch eine spitze Nadel und sind fast immer bereit, dies auch zu honorieren.

\section{Delegation}

In der Vorbehandlung und später der UPT kann bereits das Messen der Taschentiefe als unangenehm empfunden werden, ebenso die professionelle Zahnreinigung, erst recht wenn zusätzlich subgingival klinisch erreichbare Konkremente und der Biofilm entfernt werden sollen. Da es sich um keine Injektion handelt, kann Dynexan Mundgel ${ }^{\circ}$ aus Zylinderampullen auch von der qualifizierten Mitarbeiterin verwendet werden, die auch antimikrobielle Medikamente bis zum Sulkusboden applizieren darf. Griffbereit vorbereitet, kann dies ohne großen Zeitaufwand geschehen. Die Wirkung tritt schnell ein und es kann ein wesentlich entspannterer Patient weiterbehandelt werden. Natürlich kann dies abgerechnet werden, wie bei jeder anderen delegierten Leistung auch. Ausführliche Hinweise dazu finden Sie in dem Dynexan Mundgel ${ }^{\bullet}$ Zylinderampullen Beratungspaket der Firma Kreussler Pharma.

Informieren Sie sich ausführlich, fordern Sie das Musterund Beratungspaket an mit ausführlichen Hinweisen zur Abrechnung und Delegation unter der Faxnummer 0611 - 9271111 (nur so lange der Vorrat reicht)

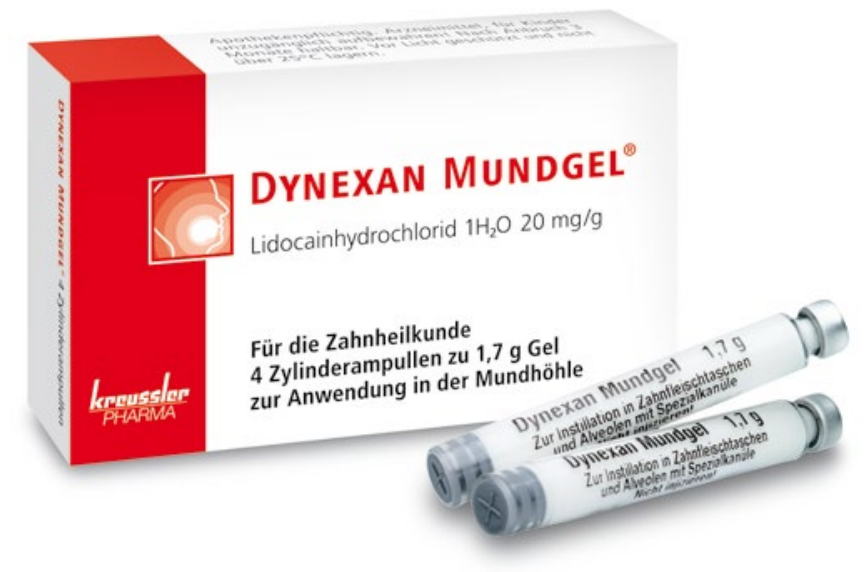

Dynexan Mundgel Zus.: $1 \mathrm{~g}$ Gel enth.: Wirkstoff: Lidocainhydrochlorid 1H2O 20 mg; weit. Bestandteile: Benzalkoniumchlorid, Bitterfenchelöl, Glycerol, Guargalaktomannan, Minzöl, dickfl. Paraffin, Pfefferminzöl, Saccharin-Natrium, hochdisp. Siliciumdioxid, Sternanisöl, Thymol, Titandioxid, weißes Vaselin, ger. Wasser. Anw.: Zur zeitweiligen, symptomatischen Behandlung von Schmerzen an Mundschleimhaut, Zahnfleisch und Lippen. Gegenanz:: absolut: Überempfindl. gegenüber den Inhaltsst. von Dynexan Mundgel od. gegen and. Lokalanästhetika vom Säureamid-Typ. relativ: Pat. mit schweren Störungen d. Reizbildungs- $u$. Reizleitungssystems am Herzen, akuter dekompensierter Herzinsuffizienz u. schweren Nieren- od. Lebererkrankungen. Nebenw: Sehr selten ( $<0,01 \%$ einschl. Einzelfälle): lokale allerg. und nichtallerg. Reakt. (z. B. Brennen, Schwellungen, Rötungen, Jucken, Urtikaria, Kontaktdermatitis, Exantheme, Schmerzen), Geschmacksveränd., Gefühllosigkeit, anaphylakt. Reakt. u. Schockreakt. mit begleitender Symptomatik. Stand: 07/2015. Chemische Fabrik Kreussler \& Co. GmbH D - 65203 Wiesbaden 